\title{
AVALIAÇÃO DO CURRÍCULO DA GRADUAÇÃO EM ENFERMAGEM POR EGRESSOS
}

\author{
Débora Dada Martinéli1, Camila Rumi de Mouraª, Claudia Bernardi Cesarino², Lúcia Marinilza Beccaria², \\ Maria Helena Pinto' ${ }^{2}$ Vânia Del Arco Paschoal ${ }^{3}$
}

\begin{abstract}
RESUMO: Estudo de caso com objetivo de verificar a opinião dos egressos do Curso de Graduação, sobre dois currículos distintos, quanto à contribuição na sua formação. Para coleta de dados, realizada de novembro a dezembro de 2009, utilizou-se questionário com perguntas abertas e fechadas enviado, enviando por e-mail ou carta registrada aos 231 egressos do Currículo Mínimo de 2004 e 2005 e do Currículo Vigente de 2006 e 2007. Dos 231 egressos, 39,4\% participaram; a maioria cursou pós-graduação, atuava na área hospitalar; consideraram a sistematização da assistência de enfermagem como conteúdo essencial; avaliaram o currículo como flexível e com disciplinas integradas. Percebeu-se mudanças de concepção e práticas pedagógicas, maior integração das disciplinas básicas com atividades profissionalizantes, aumento da atividade de pesquisa e extensão, maior enfoque nas ações de promoção da saúde e prevenção da doença. Contudo, as mudanças não foram suficientes para atender às demandas das políticas públicas.
\end{abstract}

Palavras-chave: Educação em enfermagem; Currículo; Avaliação educacional; Bacharelado em enfermagem.

\section{EVALUATION OF THE COURSE CONTENT OF THE NURSING DEGREE BY ITS GRADUATES}

ABSTRACT: A case study carried out with the objective of verifying the opinion of graduates of the degree in Nursing on the contribution of two different curriculums to their professional formation. Data was collected in November and December 2009, using a questionnaire with open and closed questions, sent either by email or registered post to the 231 former students of the 'minimum' curriculum of 2005 and 2005, and the present curriculum of 2006 and 2007. Of the 231 former students, $39.4 \%$ replied, the majority of whom were pursuing a post-graduate qualification and working in hospitals. They considered the systematization of nursing assistance to be essential content, and they evaluated the course as flexible and with integrated disciplines. The following were noted: changes in how students conceived of subjects, pedagogical practices, greater integration of basic disciplines with professionalizing activities, an increase in research activities and continuing professional development, and greater focus on health promotion and the prevention of disease. Nevertheless, the changes were not sufficient to meet the demands of public policy.

KEYWORDS: Nursing Education; Curriculum; Educational evaluation; Baccalaureate in nursing.

\section{EVALUACIÓN DEL CURRÍCULO DE LA GRADUACIÓN EN ENFERMERÍA POR EGRESOS}

RESUMEN: Estudio de caso cuyo objetivo fue verificar la opinión de los egresos del Curso de Graduación, acerca de dos currículos distintos, cuanto a la contribucción en su formación. Para recoger los datos, de noviembre a diciembre de 2009 , fue utilizado cuestionario con preguntas abiertas y cerradas por medio de e-mail o carta registrada a los 231 egresos del Currículo Mínimo de 2004 y 2005 y del Currículo Vigente de 2006 y 2007. De los 231 egresos, 39,4\% participaron; la mayoría hizo posgrado y actuava en el área hospitalar; consideraron la sistematización de la asistencia de enfermería como contenido esencial; evaluaron el currículo como flexible y con disciplinas integradas. Se percibieron cambios de concepción y prácticas pedagógicas, mayor integración de las disciplinas básicas con actividades profesionalizantes, aumento de la actividade de investigación y extensión, mayor enfoque en las acciones de promoción de la salud y prevención de enfermedad. Sin embargo, los cambios no fueron suficientes para atender a las demandas de las políticas públicas.

PALABRAS-CLAVE: Educación en enfermería; Currículo; Evaluación educacional; Graduación en enfermería.

${ }^{1}$ Acadêmica de Enfermagem da Faculdade de Medicina de São José do Rio Preto - FAMERP.

${ }^{2}$ Enfermeira. Doutora em Enfermagem. Professora do Departamento de Enfermagem Geral da FAMERP.

${ }^{3}$ Enfermeira. Doutora em Enfermagem. Professora do Departamento de Enfermagem em Saúde Coletiva e Orientação Profissional da FAMERP. 


\section{INTRODUÇÃO}

O ensino de Enfermagem no Brasil passou por diversas fases de desenvolvimento, tendo como reflexo o contexto histórico da Enfermagem e da sociedade brasileira. O perfil do enfermeiro tem exigido transformações com o quadro político-econômico-social da educação e da saúde no país e no mundo. No Brasil, a Enfermagem profissional foi introduzida em 1923, pelo Decreto n. 16300/23, no Rio de Janeiro, mediante a organização do Serviço de Enfermeiras do Departamento Nacional de Saúde Pública (DNSP), então dirigida por Carlos Chagas e posteriormente denominado Escola Anna Nery. Desde sua criação, os currículos de 1923, 1949, 1962 e 1972 foram centrados no indivíduo/ doença/cura e na assistência hospitalar ${ }^{(1)}$.

Por meio do parecer do Conselho Federal de Enfermagem n. 163/72, o currículo mínimo de Enfermagem foi reformulado, criando-se as habilitações em Saúde Pública, Enfermagem Médico-cirúrgica e Obstetrícia para serem cursadas de forma optativa, nos dois últimos semestres do curso $^{(2)}$. O Ministério da Educação e do Desporto, por meio da Portaria n. 1721 de 15 de dezembro de 1994, estabeleceu um novo currículo mínimo para o Curso de Enfermagem e definiu o perfil do enfermeiro generalista, com formação para atuar em quatro áreas: assistência, gerência, ensino e pesquisa, mas com visão de assistência holística ${ }^{(3)}$.

A premência por mudanças no campo da formação de profissionais da saúde, a partir de uma construção coletiva que buscou integrar a educação com a saúde, foi incorporada nas Diretrizes Curriculares Nacionais (DCN) dos cursos da área da saúde sob o Parecer CNE/ CES n. 1.133/2001 ${ }^{(4)}$. Com a promulgação da Lei de Diretrizes e Bases da Educação Nacional, Lei n. 9.394/96, foi prevista uma reestruturação dos cursos de graduação, com a extinção dos currículos mínimos e a adoção de diretrizes curriculares específicas para cada curso ${ }^{(1)}$.

As novas diretrizes curriculares para a Graduação em Enfermagem buscam, entre outros aspectos, levar o aluno a aprender a aprender, o que engloba aprender a ser, aprender a fazer, aprender a viver juntos e aprender a conhecer ${ }^{(4)}$. Essas diretrizes têm norteado as mudanças na formação do enfermeiro, requerendo uma formação mais crítica, flexível, versátil, reflexiva e que encaminhe soluções para os desafios da atenção à saúde da comunidade, atuando como promotor da saúde integral fundamentado nos princípios da Reforma Sanitária Brasileira e do Sistema Único de Saúde ${ }^{(5)}$.

O conjunto disposto nas DCN aborda o perfil do formando, egresso/profissional, as competências e habilidades gerais e específicas para a formação do enfermeiro, os conteúdos essenciais para o curso, a obrigatoriedade do estágio supervisionado, as atividades complementares, além de incisos que fazem referência ao projeto político-pedagógico, à organização do curso, ao acompanhamento e à avaliação ${ }^{(4)}$. No entanto, o perfil do profissional a ser formado depende do projeto pedagógico deve nascer da própria realidade, ser exequível, prever as condições de avaliação e ser uma ação articulada de todos os envolvidos da instituição de ensino e construída continuamente. O projeto pedagógico, que deve iniciar-se com a elaboração do marco referencial, sendo este a orientação de todas as suas etapas ${ }^{(6)}$.

A motivação para a mudança curricular tem como compromisso atender as necessidades de saúde da população, que precisam de cuidados profissionais de enfermagem para produzir uma melhoria efetiva na qualidade da assistência prestada, e como forma de obter o reconhecimento da sociedade do seu valor social no cuidado humano ${ }^{(7)}$. No desejo de formar profissionais competentes, no ano de 2000, constituiu-se o Núcleo de Apoio ao Ensino de Enfermagem da Faculdade de Medicina de São José do Rio Preto (FAMERP), com a finalidade de estudar e planejar a mudança curricular do Curso de Graduação em Enfermagem ${ }^{(8)}$.

Em março de 2003 iniciou-se a implantação do novo currículo para os ingressantes no Curso de Graduação em Enfermagem da FAMERP, que implicou no aumento da carga horária em Saúde Pública, formação do enfermeiro generalista com enfoque interdisciplinar, implantação da sistematização da assistência de enfermagem fundamentada no processo saúde-doença em todos os ciclos de vida do ser humano, utilização de metodologia pedagógica ativa, flexibilização do currículo com oferecimento de disciplinas optativas, implantação do estágio supervisionado nos dois últimos semestres da graduação e a realização do Trabalho de Conclusão de Curso $^{(8)}$.

Desta forma, este estudo objetivou verificar a opinião dos egressos do Curso de Graduação em Enfermagem da FAMERP sobre dois currículos distintos, quanto à contribuição na sua formação profissional, oferecendo ajustes necessários para melhoria da qualidade das atividades desenvolvidas.

\section{METODOLOGIA}

Essa pesquisa se caracteriza por ser um estudo de caso prospectivo, foi realizado com 91 egressos, formados no período de 2004 a 2005, conforme o Currículo Mínimo (CM) regido pela Portaria MEC n. 1721/94 e 
os egressos do período de 2006 a 2007 referentes ao Currículo Vigente (CV) regido pela Resolução CNE/ CES n. 3/01 do Parecer CNE/CES n. 1.133/2001, que corresponde à mudança curricular.

Os critérios de inclusão na pesquisa foram: ser exaluno do Curso de Graduação em Enfermagem da FAMERP, formado nos período citados; aceitar participar da pesquisa mediante a assinatura do Termo de Consentimento Livre e Esclarecido, sendo que o único critério de exclusão foi não responder o questionário enviado.

O instrumento de coleta de dados foi um questionário com perguntas abertas e fechadas sobre dados de identificação pessoal, inserção no mercado de trabalho, pontos fortes e fracos da mudança curricular, contribuição da formação acadêmica na atuação profissional e satisfação geral. Os dados foram coletados no ano de 2009. Inicialmente, foi feito levantamento junto à secretaria do Curso de Enfermagem, dos endereços e nomes dos formandos de 2004 a 2007.

A questão sobre satisfação geral em relação à sua formação na FAMERP apresentava uma escala de Likert 0 a 5 (incluindo os decimais), utilizada para que os egressos classificassem sua satisfação. Em seguida, os questionários foram enviados por e-mail ou mala direta com envelope-resposta selado em anexo, para que este retornasse aos pesquisadores sem causar ônus aos participantes, e impedindo a identificação do respondente.

A metodologia adotada foi a avaliação responsiva, processo com origem na distinção entre as avaliações somativa e formativa, cujo foco central é análise crítica de uma instituição, programa ou projeto independentemente de seus objetivos, mas que permite a identificação dos pontos fortes e fracos ${ }^{(9)}$. A abordagem responsiva, é uma forma diferente de focalizar uma avaliação em relação aos seus parâmetros e limites, pois nos modelos tradicionais estes são definidos a priori. Já na avaliação responsiva os parâmetros e limites são determinados por meio de um processo interativo e de negociação que abrange grupos de interesse e que desprende de uma importante parcela de tempo e recursos ${ }^{(10)}$.

As unidades de análise foram as disciplinas, estágios, ensino clínico, conteúdos, estratégias, carga horária, recursos e avaliações, permitindo assim, a identificação dos pontos fortes e fracos da mudança curricular e levando ou favorecendo subsídios para o aperfeiçoamento das mudanças implantadas. Esta pesquisa, atendendo aos procedimentos previstos na Resolução n. 196/96, do Conselho Nacional de Saúde, foi aprovada pelo Comitê de Ética em Pesquisa da FAMERP, sob protocolo n. 5572/2009.

\section{RESULTADOS}

De 231 questionários enviados, que correspondiam ao número total de egressos, obteve-se o retorno de 91(39,4\%) enfermeiros graduados na instituição, sendo $40(43,9 \%)$ do currículo mínimo e 51(56,1\%) do currículo vigente. Dos 91 egressos respondentes, $7(7,7 \%)$ foram do sexo masculino e $84(92,3 \%)$ do sexo feminino. Das 84 mulheres, 39(46,4\%) eram formadas pelo currículo mínimo (CM) e 45(53,6\%) pelo currículo vigente $(\mathrm{CV})$.

No que tange à faixa etária, a média (desviopadrão) de idade dos egressos referentes ao $\mathrm{CM}$, foi de $25,7( \pm 1,9)$ e ao CV foi de $24,1( \pm 2,8)$ anos de idade. Quanto ao tempo de inserção no mercado de trabalho, verificou-se que a maioria $(76 ; 83,5 \%)$ conseguiu seu primeiro emprego em até um ano após sua formação, sendo 38 (50\%) do CM e 38 (50\%) do CV.

Em relação ao primeiro emprego, 25(27,47\%) optaram por trabalhar em sua área de preferência e dentre estes, 10(40\%) são correspondentes ao CM e 15(60\%) ao CV; dos 91 participantes, 56(61,54\%) aproveitaram a primeira oportunidade, independentemente da área desejada. Entre as áreas de atuação, 38(41,8\%) trabalhavam em hospitais, sendo $18(47,37 \%)$ formados pelo $\mathrm{CM}$ e 20(52,63\%) pelo CV; $28(30,8 \%)$ atuaram em saúde pública, e dentre estes $13(46,43 \%)$ eram do $\mathrm{CM}$ e $15(53,57 \%)$ do CV; e ainda, $27,4 \%$ do total de respondentes atuaram em outras áreas como empresa, ensino/educação e pesquisa.

Quanto à atividade realizada, 64(70,3\%) afirmaram ser: na assistência, sendo $31(49 \%)$ do CM e 33(51\%) do $\mathrm{CV}$; na gerência $11(12,1 \%)$, sendo $8(72,7 \%)$ do $\mathrm{CM}$ e $3(27,3 \%)$ do $\mathrm{CV}$; no ensino foram $14(15,4 \%)$, sendo $8(57 \%)$ do CM e $6(43 \%)$ do CV. Do total de participantes apenas 2(2,2\%) eram pesquisadores na área de Enfermagem e estes foram formados pelo CV (100\%), sendo que entre os formados pelo $\mathrm{CM}$ nenhum era pesquisador.

Sobre a realização de pós-graduação, $73(80,2 \%)$ já haviam cursardo ou estavam cursando especialização (lato sensu), sendo 38 (52\%) do CM e 35(48\%) do CV, e $8(8,8 \%)$ eram pós-graduandos em stricto sensu, sendo $3(37,5 \%)$ do CM e 5(62,5\%) do CV.

Dos participantes, $79(86,8 \%)$ relataram ter aprendido a aplicar adequadamente a Sistematização da Assistência de Enfermagem (SAE) na graduação, dos quais $33(41,8 \%)$ eram do CM e 46(58,2\%) do CV. A SAE foi considerada disciplina essencial para o exercício da profissão por 43 egressos $(47,25 \%)$, correspondendo a $12(28 \%)$ do $\mathrm{CM}$ e $31(72 \%)$ do $\mathrm{CV}$. 
Em relação às disciplinas consideradas essenciais, $21(23 \%)$ afirmaram que todas eram necessárias para o exercício da profissão, sendo $6(28,6 \%)$ referentes do CM e 15(71,4\%) do CV. Também foram citados o estágio supervisionado e as disciplinas optativas pelos egressos formados pelo $\mathrm{CV}$.

No que se refere à carga horária prática do curso, dos 51 respondentes do CV, 25(49\%) consideraram ser suficiente para adquirir aptidão para a prática profissional, enquanto que dos 40 participantes do CM, 26(65\%) consideraram a carga horária suficiente. Em relação à carga horária teórica, 33(82,5\%) do CM e 37(72,5\%) do $\mathrm{CV}$ afirmaram ser satisfatória para o aprendizado.

Os conteúdos teóricos e práticos ministrados na graduação foram considerados aplicáveis na prática profissional por $88(96,7 \%)$ dos respondentes, sendo $40(45,5 \%)$ do $\mathrm{CM}$ e $48(54,5 \%)$ do CV. A maioria dos egressos, 80(87,9\%) deles, acreditava que o currículo da FAMERP ofereceu disciplinas de forma integrada, sendo 34(42,5\%) do $\mathrm{CM}$ e $46(57,5 \%)$ do CV. Sobre os preceitos éticos e humanísticos ministrados durante a graduação, $86(94,5 \%)$ consideraram relevantes ao exercício da profissão.

$\mathrm{Na}$ percepção dos graduados, o currículo da FAMERP foi considerado flexível por $60(65,9 \%)$, sendo $25(41,6 \%)$ do $\mathrm{CM}$ e $35(58,4 \%)$ do CV.

O campo de estágio foi considerado adequado para seu processo de ensino-aprendizado por $85(93,4 \%)$ dos egressos em ambos os currículos, sendo $39(46 \%)$ do CM e 46(54\%) do CV. Entre os respondentes, 48 $(52,7 \%)$ afirmaram ter faltado algo em seu aprendizado que interferiu na vida profissional, sendo $20(41,7 \%)$ do $\mathrm{CM}$ e $28(58,3 \%)$ do $\mathrm{CV}$. A média de satisfação do egresso do CM foi de 4,4 $( \pm 0,7)$ e do CV 4,2 $( \pm 0,6)$.

\section{DISCUSSÃO}

Observando-se os dois currículos, foi possível verificar que a maioria dos participantes do gênero feminino era formada pelo currículo vigente e que tanto o CM como o CV tinham predomínio de jovens, com médias de 25,7 e 24,8 anos de idade, respectivamente. A profissão de enfermeiro continua sendo eminentemente feminina, independente do estado ou região do país. Em estudo realizado em três Instituições de Ensino Superior que ministram Curso de Graduação em Enfermagem no Município de João Pessoa, no período de 2008 a 2009, 90,2\% dos participantes eram do sexo feminino, o que é justificável pelo fato do Curso de Enfermagem possuir características históricas ligadas ao gênero feminino ${ }^{(11)}$.
Em relação à inserção no mercado de trabalho, verificou-se que a maioria iniciou em menos de um ano. Também um estudo realizado com egressos das três primeiras turmas graduadas, nesta mesma instituição, observou-se que $94,3 \%$ dos egressos obtiveram seu primeiro emprego em até um ano de formado ${ }^{(12)}$.

Notou-se predomínio da inserção no mercado de trabalho na área hospitalar e centrados nas atividades assistenciais. Pesquisa realizada no Curso de Graduação em Enfermagem da Universidade Estadual de Londrina, mostrou que $72,2 \%$ dos egressos estavam na área hospitalar, seguida da Saúde Pública (14,8\%), e que 65,5\% atuavam na assistência, justificando que a formação pelo currículo mínimo era centrada na atuação hospitalar ${ }^{(13)}$. A inserção na área hospitalar foi igualmente observada em outro estudo, sendo que de 32 egressos, 26 atuavam em instituições hospitalares públicas ou privadas, seguidos por aqueles atuando em Serviços de Saúde Pública como Unidades Básicas de Saúde com Estratégia Saúde da Família ${ }^{(15)}$.

Percebe-se que o egresso de Enfermagem procura aprimorar seus conhecimentos por meio de cursos de pós-graduação lato sensu, como foi observado neste estudo, em que $80,2 \%$ cursavam ou estavam inseridos neste nível de formação. Estudo desenvolvido no Curso de Enfermagem da Universidade Estadual de Londrina, apontava que $54,4 \%$ dos egressos já tinham título de especialista ${ }^{(13)}$. Estes dados confirmam que os egressos se preocupam com uma melhor qualificação.

A maioria dos egressos relatou ter aprendido aplicar adequadamente a Sistematização da Assistência de Enfermagem durante a graduação, no entanto, observou-se que a maior porcentagem destas respostas veio dos graduados do CV. De acordo as Diretrizes e Bases da Educação o processo de enfermagem deveria estar inserido de forma mais efetiva nos currículos de Graduação em Enfermagem ${ }^{(12)}$.

O estágio curricular supervisionado se tornou obrigatório para o Curso de Graduação em Enfermagem com a instituição, em 2001, das Diretrizes Curriculares, correspondendo a uma carga horária mínima de $20 \%$ da carga horária total do curso, a ser realizado em hospital geral e especializado, ambulatório, rede básica de serviços de saúde ou comunidade. Nele, o estudante tem a oportunidade de desenvolver as quatro competências do profissional enfermeiro: cuidar, gerenciar, educar e pesquisar, vivenciando o contexto de inserção na realidade do mundo do trabalho ${ }^{(16)}$.

No presente estudo, encontrou-se maior percentual dos egressos do CV que consideravam que as disciplinas estão integradas. Isto pode ser justificado pela 
mudança curricular, na qual o processo de formação de profissionais de enfermagem deve pautar-se no desenvolvimento de ações acadêmicas, multi e interdisciplinares, com base humanista, com ética e com capacidade crítica, na perspectiva de observar a integralidade do cuidado, o que pressupõe a formação de profissionais capazes de enfrentar os problemas complexos que se apresentam na sociedade contemporânea, mais especificamente, na área da saúde ${ }^{(17)}$. Este é o objetivo da Escola de em Enfermagem da Universidade de São Paulo, que com a mudança curricular visa aumentar a integração das disciplinas e dos departamentos, a autonomia do estudante e a adoção do Sistema Único de Saúde como orientação geral do currículo ${ }^{(18)}$.

Estudo realizado com graduandos e recém-graduados de Enfermagem de uma universidade particular da Cidade de São Paulo mostrou que o cuidar em enfermagem é uma atividade essencial na prática dos participantes e que para cuidar é preciso ter uma visão holística. Sendo assim, o olhar e agir do enfermeiro deve ir além do aparente, refletindo sobre todo o exposto ${ }^{(19)}$.

Os egressos consideraram a carga horária do curso satisfatória. Estes mesmos achados foram encontrados no discurso dos egressos do Curso de Graduação em Enfermagem de um centro universitário privado, que fizeram menção à importância de ter uma carga horária maior para a disciplina de Administração, com estratégias diversificadas, dando ênfase ao desenvolvimento de competências administrativas, principalmente éticopolíticas, além de considerar a indissociabilidade entre ensino, pesquisa e extensão ${ }^{(15)}$.

Neste trabalho o grau de satisfação dos egressos, em uma escala de 0 a 5 , ficou acima de 4 , mostrandose satisfeitos com sua formação. Em estudo realizado com acadêmicos da segunda à quarta série do Curso de Enfermagem de uma universidade pública, estes se monstraram estar satisfeitos com a sua formação, de acordo com as suas necessidades e expectativas em relação ao curso ${ }^{(20)}$.

Pelo estudo, também se percebe, na prática, mudanças de concepção e práticas pedagógicas na implantação de novas modalidades pedagógicas, maior integração das disciplinas básicas com as atividades profissionalizantes, aumento das atividades de pesquisa e extensão e maior enfoque nas ações de promoção da saúde e prevenção da doença.

É importante que o aluno dê sentido à sua ação profissional, entendendo que o sujeito tem de transformar a sua prática e que isso se processa mediante ações de reflexão crítica.

\section{CONCLUSÃO}

Os resultados deste estudo possibilitaram concluir que, na opinião de seus egressos, a inserção no mercado de trabalho ocorreu, predominantemente, em até um ano após a graduação, com maior prevalência na área hospitalar e atuação em atividades assistenciais de enfermagem.

Em ambos os currículos, os egressos consideraram como pontos fortes da formação: a continuidade do aprendizado por meio de cursos de pós-graduação lato sensu, afirmando a importância da aplicação das políticas de educação permanente; acreditaram que a disciplina de SAE, ministrada durante a graduação, possibilitou sua aplicação adequada na prática profissional; os preceitos éticos e humanísticos foram referidos como relevantes ao exercício da profissão e houve uma maior flexibilidade com a implantação das disciplinas optativas. Já os egressos do currículo vigente destacaram como ponto fraco em sua formação o maior enfoque na área hospitalar, apesar do aumento da carga horária em disciplinas do campo da Saúde Pública. Os egressos de ambos os currículos consideram-se satisfeitos com a formação na FAMERP.

Conhecer a opinião dos egressos sobre o currículo que os formou é fundamental para avaliar o exercício consciente e seguro da profissão e proporcionar subsídios ao processo de gestão institucional. Consequentemente, torna-se possível a melhoria contínua do processo ensinoaprendizagem para que se consiga formar o enfermeiro com o perfil almejado. Entretanto, a mudança curricular, com incorporação das Diretrizes Curriculares Nacionais, ainda não é suficientes para instrumentalizar a prática destes egressos no cotidiano dos serviços de saúde.

Essas diretrizes incentivaram mudanças curriculares com o foco principal na atenção básica, a partir das constatações de que o modelo hegemônico centrado na doença tem encarecido o sistema e não tem apresentado resolutividade, entendendo que essas mudanças não aconteceriam de forma espontânea.

Ressalta-se, ainda, que a participação dos egressos deve ser efetiva nos processos de avaliação do currículo preconizado pelas Diretrizes Curriculares Nacionais, pois é no cotidiano profissional e na aproximação do estudante com a realidade de saúde que a formação será efetivada na direção proposta.

\section{REFERÊNCIAS}

1. Ito EE, Peres AM, Takahashi RT, Leite MMJ. O ensino de enfermagem e as diretrizes curriculares nacionais: utopia $\mathrm{x}$ realidade. Rev Esc Enferm USP. 2006;40(4):570-5. 
2. Galleguillos TGB, Oliveira MAC. A gênese e o desenvolvimento histórico do ensino de enfermagem no Brasil. Rev Esc Enferm USP. 2001;35(1):80-7.

3. Brasil. Portaria n. 1721, de 15 de dezembro de 1994. Dispõe sobre currículo mínimo e duração do curso de Enfermagem. Diário Oficial da República Federativa do Brasil, Brasília, 16 dez. 1994. Seção 1:1.

4. Ministério daEducaçãoeCultura(BR). Diretrizes curriculares nacionais do curso de graduação em enfermagem. Brasília (DF): Ministério da Educação e Cultura; 2001.

5. Nóbrega-Therrien SM, Guerreiro MGS, Moreira TMM, Almeida MI. Projeto político-pedagógico: concepção, construção e avaliação na enfermagem. Rev Esc Enferm USP. 2010;44(3):679-86.

6. Veiga IPA. Projeto político-pedagógico da escola: uma construção possível. $23^{\mathrm{a}}$ ed. Campinas: Papirus; 2001. vl.

7. Pires, D. Organização do trabalho em enfermagem: implicações no fazer e viver dos trabalhadores de nível médio. Trab Educ Saúde. 2004;2(2):311-25.

8. Faculdade de Medicina de São José do Rio Preto. Programa: avaliação da reestruturação curricular do Curso de graduação em enfermagem. São José do Rio Preto: FAMERP. [2003]. Apostila.

9. Vianna HM. Avaliação educacional: teoria, planejamento e modelos. São Paulo: Brasa; 2000.

10. Wetzel C, Kantorski LP. Evaluation of mental health services in the context of psychiatric reform. Texto \& Contexto Enferm. 2004;13(4):593-8.

11. Pereira FJR, Santos SR, Silva CC. Caracterização do professores e estudantes de enfermagem em João Pessoa - Paraíba. Cogitare Enferm. 2010;15(3):486-91.

12. Soler ZASG, Perroca MG, Santos MLSG, Santos MR. Inserção dos egressos do curso de graduação em enfermagem da Faculdade de Medicina de São José do Rio Preto no mercado de trabalho: parâmetros para avaliação da qualidade do ensino. Acta Paul Enferm. 2001;14(1):37-48.

13. Souza SND. O egresso do curso de graduação em enfermagem da Universidade Estadual de Londrina: perfil socioeconômicodemográfico, inserção no mercado de trabalho, atuação profissional e contribuição do curso [dissertação]. São Paulo(SP): Universidade de São Paulo; 2000.

14. Meira MDD, Kurcgant P. O ensino de administração na graduação: percepção de enfermeiros egressos. Texto \& Contexto Enferm. 2009;18(4):670-9.

15. CostaLM, Germano RM. Estágio curricular supervisionado na graduação em enfermagem: revisitando a história. Rev Bras Enferm. 2007;60(6):706-10.

16. Fernandes JD, Almeida Filho N, Santa Rosa DO, Pontes M, Santana N. Ensinar saúde/enfermagem numa nova proposta de reestruturação acadêmica. Rev Esc Enferm USP. 2007;41(n.esp):830-4.

17. Oliveira MAC, Veríssimo MDLOR, Puschel VA, Riesco MLG. Desafios da formação em enfermagem no Brasil: proposta curricular da EEUSP para o bacharelado em enfermagem. Rev Esc Enferm USP. 2007;41(n. esp):820-5.

18. Silva AL, Freitas MG. O ensino do cuidar na graduação em enfermagem sob a perspectiva da complexidade. Rev Esc Enferm USP. 2010;44(3):687-93.

19. Esperidião E, Munari DB. Holismo só na teoria: a trama de sentimentos do acadêmico de enfermagem sobre sua formação. Rev Esc Enferm USP. 2004;38(3):332-40. 